\title{
Glioma stem cells and their non-stem differentiated glioma cells exhibit differences in mitochondrial structure and function
}

\author{
EUN-JUNG KIM ${ }^{1,2^{*}}$, XIONG JIN ${ }^{1,2^{*}}$, OCK RAN KIM ${ }^{1,3^{*}}$, \\ SEOK WON HAM ${ }^{1}$, SUNG-HYE PARK ${ }^{3}$ and HYUNGGEE KIM ${ }^{1,2}$ \\ ${ }^{1}$ Department of Biotechnology, College of Life Sciences and Biotechnology, Korea University, Seoul 02841; \\ ${ }^{2}$ Institute of Animal Molecular Biotechnology, Korea University, Seoul 02841; ${ }^{3}$ Department of Pathology, \\ Seoul National University Hospital, College of Medicine, Seoul 03080, Republic of Korea
}

Received August 7, 2017; Accepted October 24, 2017

DOI: $10.3892 /$ or.2017.6075

\begin{abstract}
Although mitochondria play an important role in cell survival, their biological significance in differentiated and undifferentiated cells is not well known. In the present study, we compared the differences in the structure and function of mitochondria between undifferentiated cancer stem cells and differentiated cancer cells. Glioma stem cells (GSCs), when grown under serum culture conditions, demonstrated a decrease in stem cell marker expression and tumor sphere forming ability, while showing an increase in differentiated cell markers. Transmission electron microscopy analysis revealed that the number of mitochondria with distinct cristae and electron-dense matrices increased significantly in the non-stem differentiated glioma cells when compared to their undifferentiated GSCs. Bioinformatic analysis revealed that the glucose metabolic process gene signature was enriched in gene pools that had an increased number of stem cells. Additionally, qRT-PCR analysis revealed that the expression of various glucose metabolism genes was higher in GSCs than in non-stem differentiated glioma cells. Altogether, our results suggest that GSCs have immature mitochondria when compared to differentiated glioma cells. Notably, GSCs prefer a relatively higher glucose metabolism, which implies that they utilize different mitochondrial biosynthesis and metabolic pathways when compared to differentiated glioma cells.
\end{abstract}

Correspondence to: Professor Hyunggee Kim, Department of Biotechnology, College of Life Sciences and Biotechnology, Korea University, 145 Anam-Ro, Seongbuk-Gu, Seoul 02841, Republic of Korea

E-mail: hg-kim@korea.ac.kr

*Contributed equally

Abbreviations: GBM, glioblastoma; GSCs, glioma stem cells

Key words: glioblastoma, glioma stem cells, glucose metabolism, mitochondrial structure, non-stem differentiated glioma cells

\section{Introduction}

Glioblastoma (GBM) is the most common primary tumor of the central nervous system. According to the World Health Organization, it is a grade IV brain tumor exhibiting lethal malignancy with a median survival of less than 15 months, despite the availability of several anticancer regimens such as debulking surgery, radiotherapy and chemotherapy (1). In 1997, Bonnet and Dick were the first to isolate cancer stem cells (CSCs) in acute myeloid leukemia that were known to cause cancer, metastasis, cancer resistance and recurrence (2). Since then, CSCs have been found in most solid cancers $(3,6)$. Like other types of CSCs, many recent studies have reported that glioma stem cells (GSCs) are a major cause for the initiation, progression, invasion and even recurrence of GBM (7). GSCs share many biological characteristics with normal neural stem cells. For example, when grown under serum-free stem cell culture conditions with growth factors such as epithelial growth factor (EGF) and basic fibroblast growth factor (bFGF), GSCs can form spheres and self-renew to maintain stem cell properties (8). They also differentiate into non-stem differentiated glioma cells through a variety of intrinsic and extrinsic signals, which are ultimately considered to be the main cause of tumor heterogeneity (9).

The function of mitochondria is essential, not only in normal cells, but also in cancer cells (10). Mitochondrial metabolic homeostasis and glucose metabolism signaling are known to be regulated by a variety of oncogenes and tumor suppressor genes (11). Unlike cancer cells, not many studies on the role of mitochondria in CSCs are available. Nonetheless, some studies have focused on the production and release of mitochondrial energy in CSCs $(12,13)$. It has also been suggested that mitochondria play an important role in the expansion and survival of CSCs (14). Notably, many studies have shown that the number, the structural features and the functions of mitochondria were significantly elevated and maturated during the differentiation of embryonic stem cells $(15,16)$. These studies suggested that the characteristics of mitochondria could act as biomarkers for normal stem cells and CSCs. However, there have been no studies highlighting the differences in mitochondria between CSCs and their differentiated cancer cell progenies. Thus, in the present 
study, we compared the characteristics of mitochondria between GSCs and differentiated glioma cells using transmission electron microscopy, bioinformatics and glucose metabolism analyses.

\section{Materials and methods}

Cell culture and reagents. Patient-derived 528NS, 84NS, 19NS, 157NS, and 83NS GSCs were kindly provided by Dr Ichiro Nakano. GSCs were cultured under stem cell culture conditions as follows: Dulbecco's modified Eagle's medium (DMEM)/F12 (Lonza, Basel, Switzerland) supplemented with modified B27 (0.04\%; Invitrogen, Waltham, MA, USA), 1\% penicillin/streptomycin (Lonza), L-glutamine ( $2 \mathrm{mmol} / \mathrm{l})$, gentamicin sulfate $(50 \mu \mathrm{g} / \mathrm{ml}$; Mediatech, Inc., Tewksbury, MA, USA), epidermal growth factor (EGF; $20 \mathrm{ng} / \mathrm{ml}$; R\&D Systems, Minneapolis, MN, USA) and basic fibroblast growth factor (bFGF; $20 \mathrm{ng} / \mathrm{ml}$; R\&D Systems) (17). Serum culture condition was as follows: DMEM (Lonza) supplemented with 5\% fetal bovine serum (FBS; Biotechnics Research, Inc., Lake Forest, CA, USA), $1 \%$ penicillin-streptomycin (Lonza), $1 \%$ l-glutamine (Lonza) and gentamicin sulfate $(50 \mu \mathrm{g} / \mathrm{ml})$. For mitochondrial function studies, 84NS and 528NS GSCs were used because of their ability to proliferate under stem cell culture conditions. Additionally, these GSCs displayed considerable differences in cell morphology and number of mitochondria compared to the other GSCs and non-stem differentiated glioma cells.

Quantitative reverse transcription- $P C R(q R T-P C R)$. $\mathrm{qRT}$-PCR was performed to determine mRNA levels. Total RNA of GSCs was isolated using QIAzol Lysis reagent (Qiagen, Venlo, The Netherlands) according to the manufacturer's instructions. After quantitating the amount of total RNA, $1 \mu \mathrm{g}$ RNA was used as a template to synthesize cDNA by using the RevertAid First-Strand cDNA Synthesis kit (Thermo Fisher Scientific, MA, USA). qRT-PCR analysis was conducted on an iCycler IQ Real-Time detection system (Bio-Rad Laboratories, Hercules, CA, USA) by using SYBR Premix Ex Taq (Takara Bio, Shiga, Japan) according to the manufacturer's instructions. The results of qRT-PCR were collected as $\mathrm{Ct}$ value, and in turn were quantified with the $\Delta \Delta \mathrm{Ct}$ method (18). Primer sequences used for qRT-PCR amplification were as follows (5' to $3^{\prime}$ ): $18 \mathrm{~S}$ (loading control): forward (F), CAGCCACCCGAGATT GAGCA and reverse (R), TAGTAGCGACGGGCGGTGTG; CD133: F, AATTCACCAGCAACGAGTCC and R, CCGAA TCCATTCGACGATAG; SOX2: F, TGGACAGTTACGCGC ACAT and R, ACTTGACCACCGAACCCAT; GFAP: F, GG AACATCGTGGTGAAGACC and R, AGAGGCGGAGCA ACTATCCT; GLUT1: F, TCATCGTGGCTGAACTCTTC and R, ACCACACAGTTGCTCCACAT; GLUT3: F, CGTT GTTGGAATTCTGGTGGC and R, CTTAGCATTCTCCT CTTCTTTT; HK2: F, ACCTTTGTGAGGTCCACTCC and R, TGTCCGTTACTTTCACCCAA; PDK1: F: CACCAAG ACCTCGTGTTGAG and R, AGCTTCAGGTCTCCTTGG AA; LDHA: F, GCACGTCAGCAAGAGGGAGAAAG and R, AGGTAACGGAATCGGGCTGAATC; LDHB: F, ACT TTTCTTGAGCTGAGCAACC and R, TTGAGAATGAAG TCTTCCTGAGC; MCT1: F, TGTAATCTACCAGTGGTG CTC and R, AACCTACTTCTTTCCCCCATC.
Transmission electron microscopy (TEM) analysis. For TEM assay, the cells were sufficiently washed with $0.1 \mathrm{M}$ phosphate buffer ( $\mathrm{pH}$ 7.4) and harvested as a cell pellet. Then, the pellet was fixed, first with $2.5 \%$ glutaraldehyde at $4^{\circ} \mathrm{C}$ for 2 days, and next with $1 \%$ osmium tetroxide for $1 \mathrm{~h}$. Next, the pellet was dehydrated with low to high concentrations of alcohol and then placed in propylene oxide for $30 \mathrm{~min}$. Furthermore, the pellet was permeated with low to high concentrations of epoxy resin for $3 \mathrm{~h}$, and fixed in epoxy resin overnight. Finally, epoxy sections were cut at $60 \mathrm{~nm}$, attached in a grid, and stained with uranyl acetate and lead citrate. The sections were observed with a transmission electron microscope (Hitachi H-7100; Hitachi, Ltd., Tokyo, Japan).

Bioinformatic data analysis. A microarray dataset (17) (accession no.: GSE4536) obtained from the Gene Expression Omnibus (GEO) database at http://www.ncbi.nlm.nih.gov/geo/ was used to analyze the mitochondrial related gene set signatures in both the serum and stem GSC groups. These gene set signatures were also used to analyze the correlations to the mitochondrial biogenesis gene set signatures. Furthermore, Gene Ontology of these gene set signatures was analyzed by The Database for Annotation, Visualization and Integrated Discovery (DAVID) bioinformatics resources (https://david. ncifcrf.gov) $(19,20)$.

Glucose uptake assay. To measure the glucose uptake by GSCs and the serum-induced differentiated GSCs (5\% FBS for 2 weeks), the cells were cultured in DMEM (no phenol red, serum-free; Gibco, Thermo Fisher Scientific) at $37^{\circ} \mathrm{C}$. Glucose concentration in the culture medium was measured using a Glucose (GO) Assay kit (Sigma-Aldrich, St. Louis, MO, USA). Glucose consumption was determined as the difference in glucose concentration between growth and stock medium.

Lactate production assay. For the assay, GSCs and seruminduced differentiated GSCs (5\% FBS, 2 weeks) were cultured in DMEM (no phenol red, serum-free; Gibco) for the indicated time. Lactate production in the medium was detected by using the L-lactate Assay kit (Eton Bioscience, Inc., San Diego, CA, USA) according to the manufacturer's protocol.

Statistical analysis. The Student's t-test was used to analyze the statistical significance in differences between the paired groups. Data are expressed as mean \pm standard error of the mean (SEM). All statistical tests were two-sided and data with $\mathrm{P}<0.05$ or $\mathrm{P}<0.01$ were assumed to be statistically significant.

\section{Results}

Stem cell culture conditions induce tumorsphere-forming abilities and expression of stem cell markers. Many studies have reported that cell culture conditions can alter cell phenotype, genotype and characteristics $(17,21)$. We first compared the characteristics of GSCs, which were reported to change in cell culture conditions. As a result, 528NS and 84NS GSCs grew in the form of glial cells adhering to the cell culture plate in serum-supplemented cell culture conditions known to induce GSC differentiation, whereas they grew in the form of tumorspheres, a hallmark of GSCs (22), in stem cell culture 

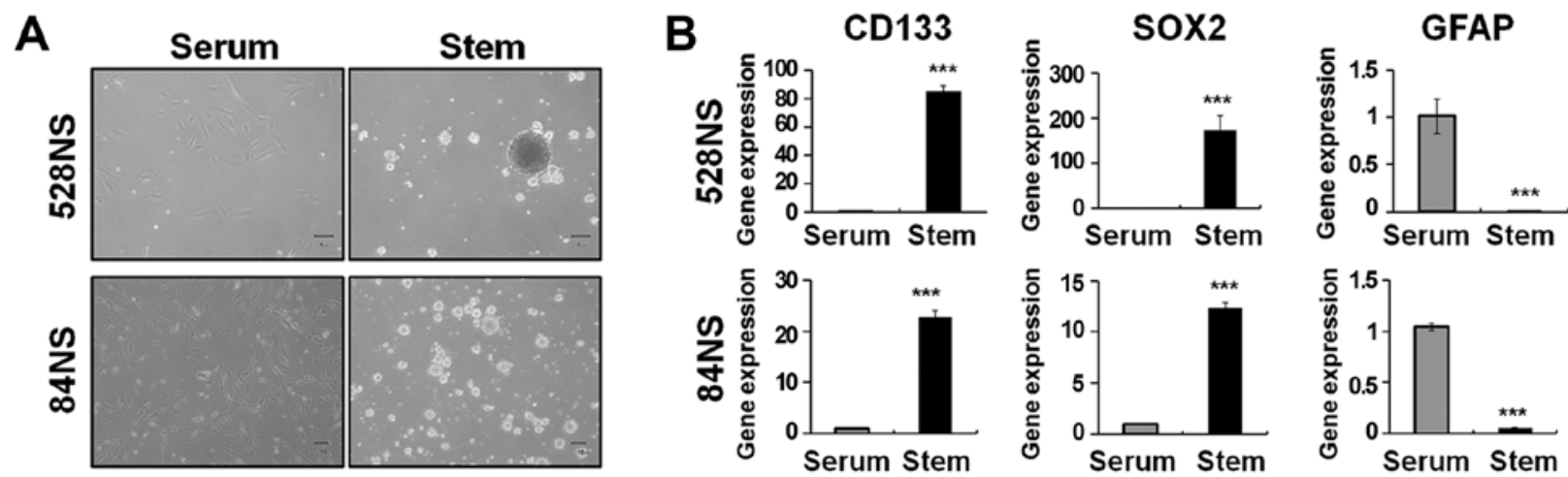

Figure 1. Changes in cell characteristics and marker gene expression in GSCs according to cell culture conditions. (A) Representative images showing morphology of 528NS and 84NS GSCs in serum-supplemented cancer cell (serum) culture and serum-free stem cell (stem) culture conditions. Scale bar, $5 \mu \mathrm{m}$. (B) Expression levels of CD133 (GSC marker), SOX2 (GSC marker), and GFAP (differentiated astrocyte marker) mRNAs in 528NS and 84NS GSCs in serum and stem culture conditions were analyzed by qRT-PCR. Data represent the mean \pm SEM $\left({ }^{* * *} \mathrm{P}<0.001\right)$.

A

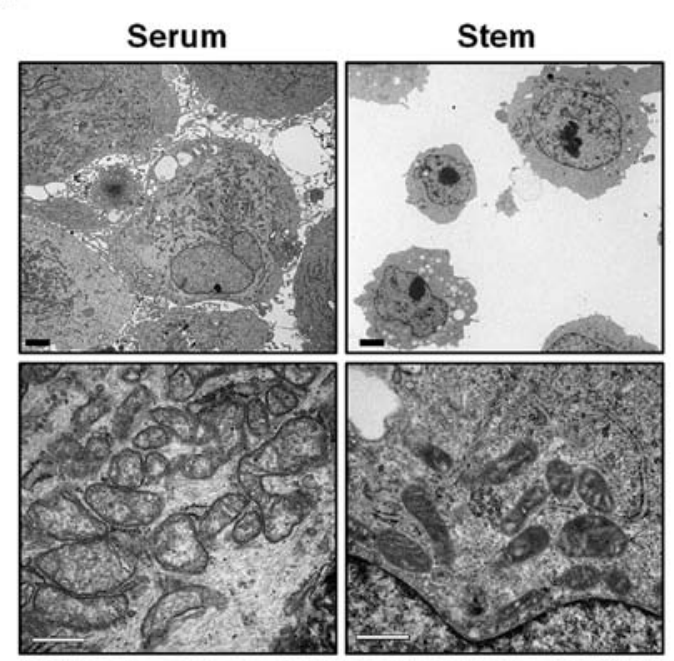

84NS

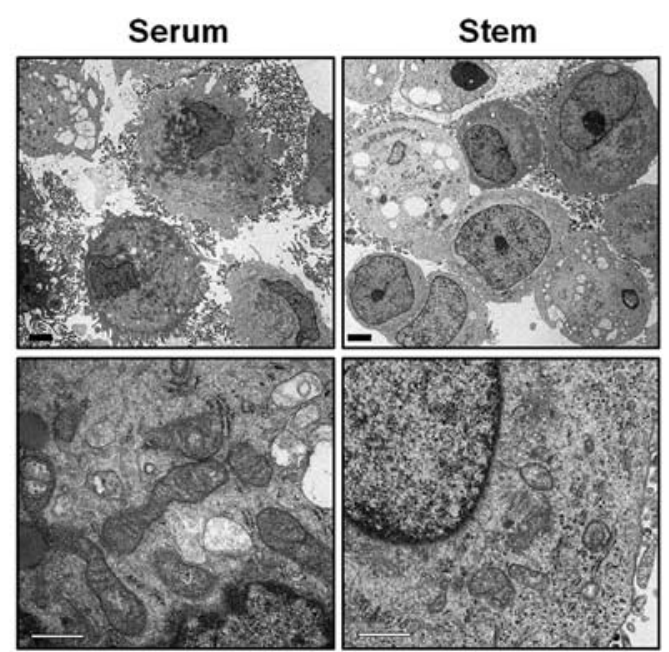

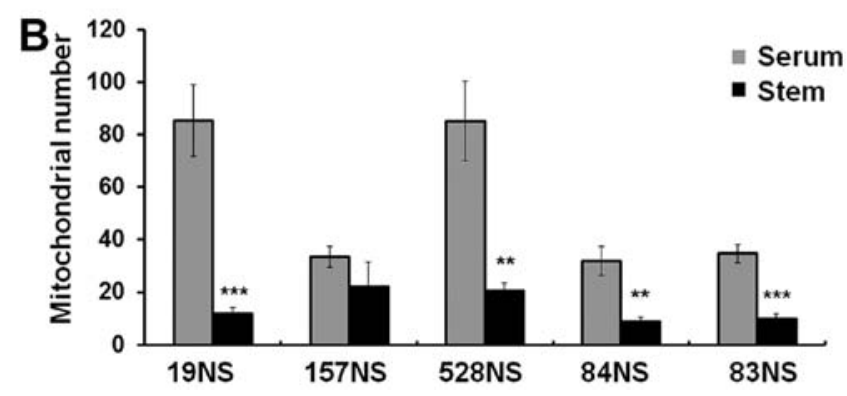

Figure 2. Suppression of mitochondrial biogenesis in GSCs grown in stem culture conditions. (A) Representative transmission electron microscopy images showing cells and mitochondrial morphology of 528NS and 84NS GSCs grown in serum and stem culture conditions. Scale bar, $2 \mu \mathrm{m}$ (black) and $500 \mathrm{~nm}$ (white). (B) The number of mitochondria in five GSCs grown in serum and stem culture conditions were detected by transmission electron microscopy analysis. Data represent the mean $\pm \operatorname{SEM}\left({ }^{* *} \mathrm{P}<0.01,{ }^{* * * *} \mathrm{P}<0.001\right)$

conditions supplemented with defined growth factors without serum (Fig. 1A). We further analyzed the mRNA expression of stem cell markers and differentiated cell markers. GSCs exhibited significantly increased expression of stem cell markers, CD133 and SOX2, and markedly decreased differentiated cell marker GFAP under stem cell culture conditions (Fig. 1B). These results suggest that GSCs exhibit different characteristics of stem cells and differentiated cells based on culture conditions.
Stem culture conditions suppress mitochondrial biogenesis in glioma stem cells. Next, we performed transmission electron microscopy analysis to determine the differences in cell structure and organelles between GSCs and their non-stem differentiated glioma cells. We found that the cell size of the differentiated GSCs was greater than that of the GSCs when visualized at a low magnification (Fig. 2A; black scale bar, $2 \mu \mathrm{m})$. Notably, when observed at high magnification, we found more mitochondria with distinct cristae and electron- 
A

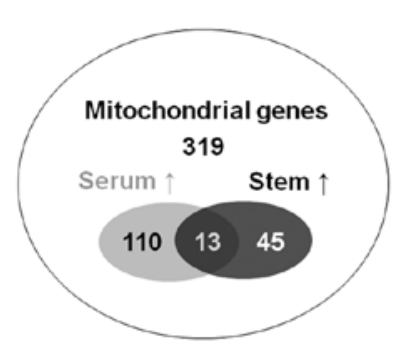

B

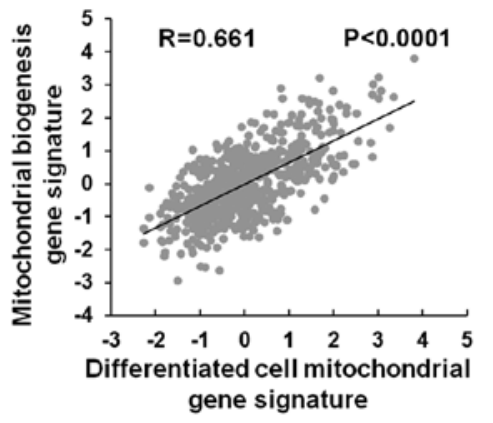

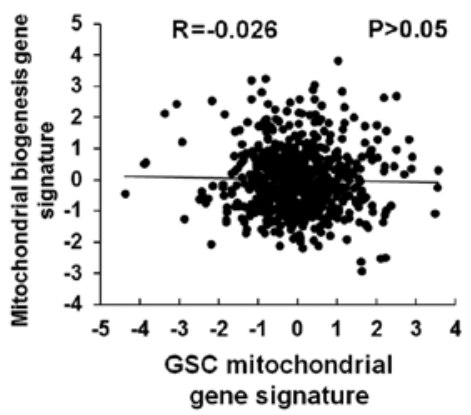

D
C

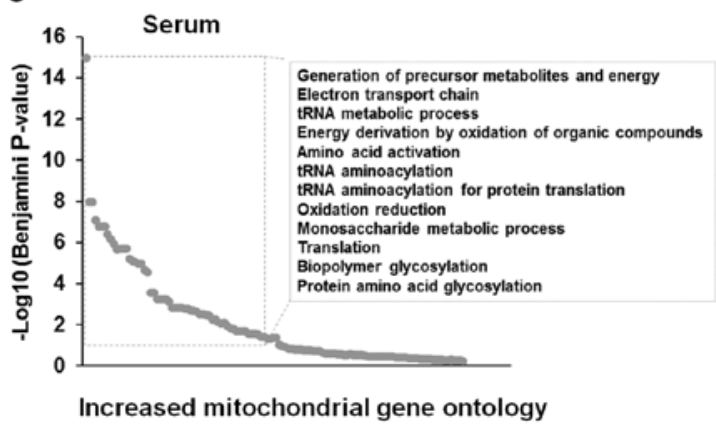

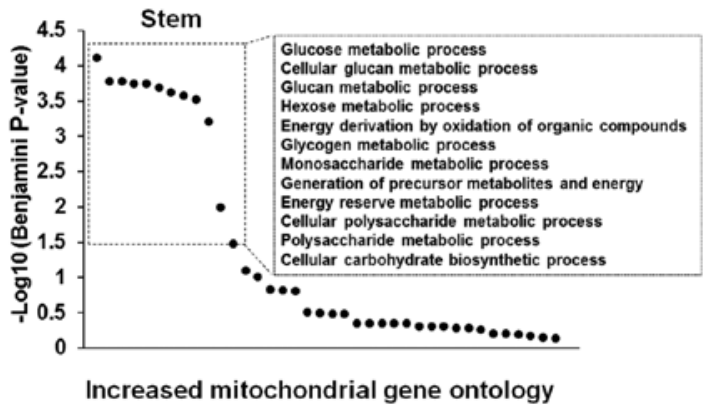

Figure 3. Bioinformatic analysis of mitochondrial genes from the transcriptome dataset of GSCs grown in serum and stem culture conditions. (A) Venn diagram showing mitochondrial genes of GSCs grown in serum and stem culture conditions presented using a previously published microarray dataset (17). (B) Comparison of the correlation between the mitochondrial biogenesis gene signature and the mitochondrial gene signature of differentiated or undifferentiated GSCs. (C) Gene Ontology analysis using the mitochondrial gene signature upregulated in non-stem differentiated glioma cells. The dots in the box indicate significant gene ontology, and the list represents the top 12 gene ontologies. (D) Gene Ontology analysis using the mitochondrial gene signature upregulated in undifferentiated GSCs. The dots in the box indicate significant gene ontology, and the list represents the top 12 gene ontologies.

dense matrices in the differentiated cells. (Fig. 2A; white scale bar, $500 \mathrm{~nm}$ ). In addition, the differences in mitochondria between these differentiated and undifferentiated GSCs were analyzed in 5 GSCs. As a result, the number of mitochondria were significantly lower in 4 out of 5 GSCs when compared to their non-stem differentiated glioma cells (Fig. 2B). These results suggest that undifferentiated GSCs may have different mitochondrial functions and metabolic energy systems when compared with differentiated glioma cells.

The gene signature for the glucose metabolic process was enriched in gene pools that are increased in stem cells. To investigate the effect of mitochondrial differences in GSCs and differentiated glioma cells, we analyzed the differentially expressed genes (DEGs) using published microarray datasets (17). Of the 319 mitochondrial-related genes, 110 genes were specifically upregulated in non-stem differentiated glioma cells, while 45 genes were upregulated in GSCs (Fig. 3A). We also determined Pearson's correlation coefficient using these gene sets and the mitochondrial biogenesis gene signature. As a result, mitochondrial biogenesis gene signature showed a significant positive correlation with the mitochondrial-related gene signatures of differentiated glioma cells $(\mathrm{R}=0.661, \mathrm{P}<0.0001)$, but not with that of GSCs $(\mathrm{R}=-0.026, \mathrm{P}>0.05)$ (Fig. 3B). These results indicate that the expression of mitochondrial biogenesis and mitochondrialrelated genes in differentiated and undifferentiated GSCs tend to be similar to mitochondria number and maturity in these cells (as shown in Fig. 2).
Next, we analyzed the Gene Ontology to examine specific cellular functions associated with mitochondrial-related gene signatures in differentiated and undifferentiated GSCs. As a result, distinct differences were observed between the mitochondrial-related gene signatures of these cells. The most obvious gene ontologies of the mitochondrial-related gene signature upregulated in non-stem differentiated glioma cells included the generation of precursor metabolites and energy, electron transport chain and various anabolic processes (Fig. 3C). However, most of the top 12 gene ontologies of the mitochondrial-related gene signature upregulated in GSCs were associated with the metabolic processes of glucose, glucan, hexose, monosaccharide and polysaccharide (Fig. 3D). Altogether, these results suggest that the mitochondrial-mediated energy production plays an important role in the growth of differentiated glioma cells. However, an effective glucose metabolism may be important for maintaining GSC characteristics even though it is regulated without fully functional mitochondria.

Glucose consumption, lactate production and glycolysisrelated genes are upregulated in GSCs grown in serum-free stem cell culture conditions. To confirm the significance of the mitochondrial gene ontologies upregulated in GSCs when compared to non-stem differentiated glioma cells, we examined the activation of the glycolytic metabolic processes, such as glucose uptake, lactate production and the expression of genes related to glycolysis in GSCs. We found that GSCs showed increased glucose consumption than differentiated 

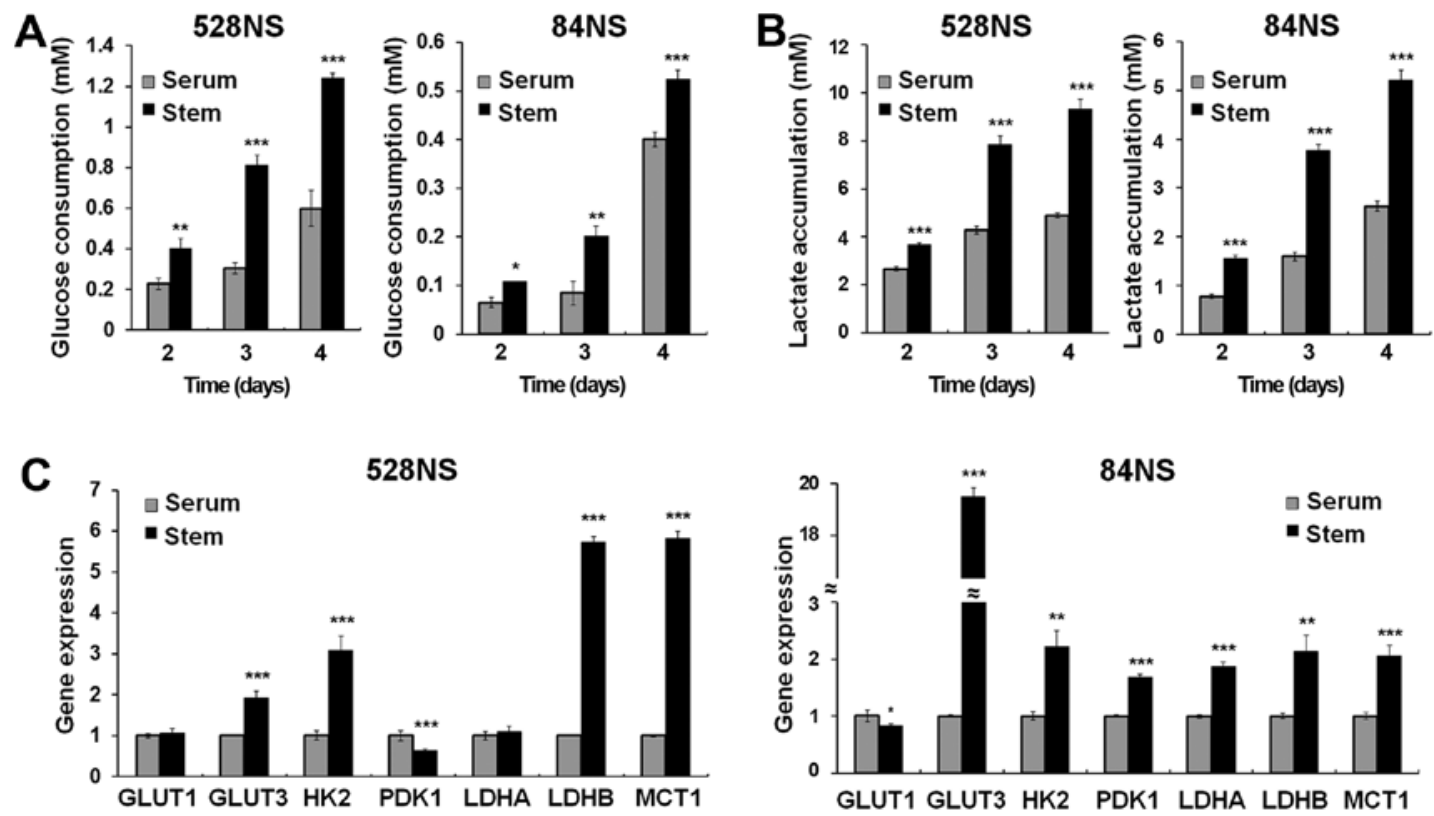

Figure 4. Glucose metabolism and glycolytic gene expression in differentiated and undifferentiated GSCs. (A) Glucose consumption measured at indicated times in 528NS and 84NS GSCs grown in serum and stem culture conditions. The data represents mean $\pm \mathrm{SEM}\left({ }^{*} \mathrm{P}<0.05,{ }^{* * *} \mathrm{P}<0.01,{ }^{* * * *} \mathrm{P}<0.001\right)$. (B) Lactate accumulation detected at indicated times in 528NS and 84NS GSCs grown in serum and stem culture conditions. The data represents mean \pm SEM $\left({ }^{* * *} \mathrm{P}<0.001\right)$. (C) Relative mRNA expression levels of glycolytic genes were determined by qRT-PCR. GLUT1, glucose transporter 1; GLUT3, glucose transporter 3; HK2, hexokinase 2; PDK1, pyruvate dehydrogenase kinase 1; LDHA, lactate dehydrogenase A; LDHB, lactate dehydrogenase B; MCT, monocarboxylate transporter. Data represent mean $\pm \mathrm{SEM}\left({ }^{*} \mathrm{P}<0.05,{ }^{* *} \mathrm{P}<0.01,{ }^{* * *} \mathrm{P}<0.001\right)$.

GSCs (Fig. 4A). The accumulation of lactate (byproduct of glycolysis) also increased in GSCs than in differentiated glioma cells (Fig. 4B). Furthermore, qRT-PCR analysis also revealed that various glycolytic enzymes such as GLUT3, HK2, LDHB and MCT1 exhibited increased expression levels in GSCs when compared to differentiated glioma cells (Fig. 4C). Altogether, these results suggest that differences in culture conditions alter mitochondrial characteristics and glucose metabolism of undifferentiated GSCs and differentiated glioma cells.

\section{Discussion}

Similar to other tumors, one of the biggest obstacles in treating GBM is tumor recurrence due to tumor heterogeneity (23). Tumor heterogeneity is the presence of various cancer and stromal cells in the tumor. Undifferentiated CSCs, which are inherently resistant to anticancer drugs, are the new, attractive target for the development of anticancer drugs as they have been identified as the main cause for the recurrence of cancer even after anticancer treatment $(24,25)$.

Recently, cancer cell metabolism has been intensively studied in various human malignancies and the metabolism of CSCs has been found to be relatively inadequate (26). Mitochondria act as factories for energy and cellular metabolite production. Mitochondrial function has also been shown to play an important role in cancer initiation, progression, metastasis, and resistance to anticancer drugs, all of which are characteristics of CSCs $(27,28)$. Therefore, understanding the nature of mitochondria in CSCs is an important step in the development of new anticancer agents.

In the present study, we found that the most distinctive feature of GSCs when compared to their non-stem differentiated progenies was the morphology and number of mitochondria. GSC mitochondria are small in size and low in number, lack well-defined cristae and electron-transparent matrices and exhibit a punctate and perinuclear arrangement. Some of these properties have been reported in other normal embryonic stem cells, induced pluripotent stem cells and in some CSCs $(29,30)$. However, this study differs from previous ones in that we clearly identified the structural and functional metabolic differences in mitochondria by using undifferentiated GSCs and their non-stem differentiated glioma cells. However, further studies are required to clarify whether these differences in mitochondria in these cells are due to differences in differentiation and undifferentiated cells or differences in cell culture conditions.

One of the most prominent expression differences in mitochondrial-related gene signatures between GSCs and non-stem differentiated glioma cells was aerobic glycolysis. In many previous studies, cancer cells were shown to be more active in aerobic glycolysis than normal cells (31), but our results indicated that GSCs were more active than cancer cells in promoting lactate secretion by aerobic glycolysis possibly owing to increased glucose uptake and immature mitochondria. Our results highlighting the differences in glucose metabolism between GSCs and non-stem differentiated glioma cells are of particular relevance in cancer biology. In terms of energy production, compared to non-malignant (or normal) cells, cancer cells should be more effective in activating the tricarboxylic acid (TCA) cycle; however, they potentially utilize aerobic glycolysis and pentose phosphate pathway to a larger extent for producing essential building blocks for cells, such as nucleic acids and lipids (32-34). It is also known that cancer cells increase lactate production via active aerobic glycolysis. This promotes changes in the tumor microenvironment, 
which increases the recruitment of M2-type macrophages and promotes cancer cell malignancy $(35,36)$. However, our results demonstrated that GSCs possessed more active pathways for aerobic glycolysis and lactate production than the non-stem differentiated glioma cells. We believe that this phenomenon of GSCs is not responsible for the aforementioned proliferation or tumor microenvironment changes. Similar to normal stem cells, GSCs likely utilize active aerobic glycolysis as an effective way to maintain stem cell fidelity by reducing genetic instability via reduction of reactive oxygen species produced in the TCA cycle due to immature mitochondria (37). In GSCs, immature mitochondria or aerobic glycolysis can be regulated by the same or similar mechanisms as those involved in stemness regulation. One such stemness signaling cue is Myc, which simultaneously regulates stemness and aerobic glycolysis. However, in future research, this hypothesis needs to be substantiated $(38,39)$.

\section{Acknowledgements}

We are grateful to all the members of the Cell Growth Regulation Laboratory for their valid input and technical assistance. The present study was supported by grants from the National Research Foundation (NRF) funded by the Ministry of Science, ICT and Future Planning (2015R1A5A1009024 and 2017M3A9A8031425), from the Next-Generation Biogreen 21 Program (PJ01107701) and from the Korea University.

\section{References}

1. Weller M, Cloughesy T, Perry JR and Wick W: Standards of care for treatment of recurrent glioblastoma - are we there yet? Neuro Oncol 15: 4-27, 2013.

2. Bonnet D and Dick JE: Human acute myeloid leukemia is organized as a hierarchy that originates from a primitive hematopoietic cell. Nat Med 3: 730-737, 1997.

3. Singh SK, Hawkins C, Clarke ID, Squire JA, Bayani J, Hide T, Henkelman RM, Cusimano MD and Dirks PB: Identification of human brain tumour initiating cells. Nature 432: 396-401, 2004.

4. Al-Hajj M and Clarke MF: Self-renewal and solid tumor stem cells. Oncogene 23: 7274-7282, 2004.

5. O'Brien CA, Pollett A, Gallinger S and Dick JE: A human colon cancer cell capable of initiating tumour growth in immunodeficient mice. Nature 445: 106-110, 2007.

6. Zhang S, Balch C, Chan MW, Lai HC, Matei D, Schilder JM, Yan PS, Huang TH and Nephew KP: Identification and characterization of ovarian cancer-initiating cells from primary human tumors. Cancer Res 68: 4311-4320, 2008.

7. Reya T, Morrison SJ, Clarke MF and Weissman IL: Stem cells, cancer, and cancer stem cells. Nature 414: 105-111, 2001.

8. Wen PY and Kesari S: Malignant gliomas in adults. N Engl J Med 359: 492-507, 2008.

9. Blanpain C and Fuchs E: Stem cell plasticity. Plasticity of epithelial stem cells in tissue regeneration. Science 344: 1242281, 2014.

10. Friedman JR and Nunnari J: Mitochondrial form and function. Nature 505: 335-343, 2014.

11. Hsu PP and Sabatini DM: Cancer cell metabolism: Warburg and beyond. Cell 134: 703-707, 2008

12. Janiszewska M, Suvà ML, Riggi N, Houtkooper RH, Auwerx J, Clément-Schatlo V, Radovanovic I, Rheinbay E, Provero P and Stamenkovic I: Imp2 controls oxidative phosphorylation and is crucial for preserving glioblastoma cancer stem cells. Genes Dev 26: 1926-1944, 2012.

13. Vlashi E, Lagadec C, Vergnes L, Reue K, Frohnen P, Chan M, Alhiyari Y, Dratver MB and Pajonk F: Metabolic differences in breast cancer stem cells and differentiated progeny. Breast Cancer Res Treat 146: 525-534, 2014.

14. Lamb R, Harrison H, Hulit J, Smith DL, Lisanti MP and Sotgia F: Mitochondria as new therapeutic targets for eradicating cancer stem cells: Quantitative proteomics and functional validation via MCT1/2 inhibition. Oncotarget 5: 11029-11037, 2014.
15. Lonergan T, Bavister B and Brenner C: Mitochondria in stem cells. Mitochondrion 7: 289-296, 2007.

16. Rehman J: Empowering self-renewal and differentiation: The role of mitochondria in stem cells. J Mol Med (Berl) 88: 981-986, 2010.

17. Lee J, Kotliarova S, Kotliarov Y, Li A, Su Q, Donin NM, Pastorino S, Purow BW, Christopher N, Zhang W, et al: Tumor stem cells derived from glioblastomas cultured in bFGF and EGF more closely mirror the phenotype and genotype of primary tumors than do serum-cultured cell lines. Cancer Cell 9: 391-403, 2006.

18. Livak KJ and Schmittgen TD: Analysis of relative gene expression data using real-time quantitative PCR and the 2(-Delta Delta C(T)) Method. Methods 25: 402-408, 2001.

19. Huang W, Sherman BT and Lempicki RA: Systematic and integrative analysis of large gene lists using DAVID bioinformatics resources. Nat Protoc 4: 44-57, 2009.

20. Huang W, Sherman BT and Lempicki RA: Bioinformatics enrichment tools: Paths toward the comprehensive functional analysis of large gene lists. Nucleic Acids Res 37: 1-13, 2009.

21. Jeon HM, Jin X, Lee JS, Oh SY, Sohn YW, Park HJ, Joo KM, Park WY, Nam DH, DePinho RA, et al: Inhibitor of differentiation 4 drives brain tumor-initiating cell genesis through cyclin E and notch signaling. Genes Dev 22: 2028-2033, 2008.

22. Lathia JD, Gallagher J, Heddleston JM, Wang J, Eyler CE, Macswords J, Wu Q, Vasanji A, McLendon RE, Hjelmeland AB, et al: Integrin alpha 6 regulates glioblastoma stem cells. Cell Stem Cell 6: 421-432, 2010.

23. Frattini V, Trifonov V, Chan JM, Castano A, Lia M, Abate F, Keir ST, Ji AX, Zoppoli P, Niola F, et al: The integrated landscape of driver genomic alterations in glioblastoma. Nat Genet 45: 1141-1149, 2013.

24. Galli R, Binda E, Orfanelli U, Cipelletti B, Gritti A, De Vitis S, Fiocco R, Foroni C, Dimeco F and Vescovi A: Isolation and characterization of tumorigenic, stem-like neural precursors from human glioblastoma. Cancer Res 64: 7011-7021, 2004.

25. Kim JK, Jeon HY and Kim H: The molecular mechanisms underlying the therapeutic resistance of cancer stem cells. Arch Pharm Res 38: 389-401, 2015.

26. Sancho P, Barneda D and Heeschen C: Hallmarks of cancer stem cell metabolism. Br J Cancer 114: 1305-1312, 2016.

27. Weinberg SE and Chandel NS: Targeting mitochondria metabolism for cancer therapy. Nat Chem Biol 11: 9-15, 2015

28. Porporato PE, Payen VL, Pérez-Escuredo J, De Saedeleer CJ, Danhier P, Copetti T, Dhup S, Tardy M, Vazeille T, Bouzin C, et al: A mitochondrial switch promotes tumor metastasis. Cell Reports 8: 754-766, 2014.

29. Ye XQ, Li Q, Wang GH, Sun FF, Huang GJ, Bian XW, Yu SC and Qian GS: Mitochondrial and energy metabolism-related properties as novel indicators of lung cancer stem cells. Int J Cancer 129: 820-831, 2011.

30. Lonergan T, Brenner C and Bavister B: Differentiation-related changes in mitochondrial properties as indicators of stem cell competence. J Cell Physiol 208: 149-153, 2006.

31. Liberti MV and Locasale JW: The Warburg effect: How does it benefit cancer cells? Trends Biochem Sci 41: 211-218, 2016.

32. Nakazawa MS, Keith B and Simon MC: Oxygen availability and metabolic adaptations. Nat Rev Cancer 16: 663-673, 2016.

33. Beloribi-Djefaflia S, Vasseur S and Guillaumond F: Lipid metabolic reprogramming in cancer cells. Oncogenesis 5: e189, 2016.

34. Dang CV: Links between metabolism and cancer. Genes Dev 26: 877-890, 2012.

35. Xie H, Hanai J, Ren JG, Kats L, Burgess K, Bhargava P, Signoretti S, Billiard J, Duffy KJ, Grant A, et al: Targeting lactate dehydrogenase - a inhibits tumorigenesis and tumor progression in mouse models of lung cancer and impacts tumor-initiating cells. Cell Metab 19: 795-809, 2014.

36. Jones W and Bianchi K: Aerobic glycolysis: Beyond proliferation. Front Immunol 6: 227, 2015.

37. Prigione A, Ruiz-Pérez MV, Bukowiecki R and Adjaye J: Metabolic restructuring and cell fate conversion. Cell Mol Life Sci 72: 1759-1777, 2015.

38. Kim EJ, Kim SH, Jin X, Jin X and Kim H: KCTD2, an adaptor of Cullin3 E3 ubiquitin ligase, suppresses gliomagenesis by destabilizing c-Myc. Cell Death Differ 24: 649-659, 2017.

39. Sancho P, Burgos-Ramos E, Tavera A, Bou Kheir T, Jagust $P$, Schoenhals M, Barneda D, Sellers K, Campos-Olivas R, Graña $\mathrm{O}$, et al: MYC/PGC-1 $\alpha$ balance determines the metabolic phenotype and plasticity of pancreatic cancer stem cells. Cell Metab 22: 590-605, 2015. 